\title{
Use of information on the manufacture of samples for the optical characterization of multilayers through a global optimization
}

\author{
Jordi Sancho-Parramon, Josep Ferré-Borrull, Salvador Bosch, and Maria Christina Ferrara
}

\begin{abstract}
We present a procedure for the optical characterization of thin-film stacks from spectrophotometric data. The procedure overcomes the intrinsic limitations arising in the numerical determination of many parameters from reflectance or transmittance spectra measurements. The key point is to use all the information available from the manufacturing process in a single global optimization process. The method is illustrated by a case study of solgel applications. @ 2003 Optical Society of America
\end{abstract}

OCIS codes: $310.3840,310.6860,160.4760,160.6060$.

\section{Introduction}

The determination of optical constants of materials from spectrophotometric data is standard practice for thin-film coatings. Typically, reflectance data, transmittance data, or both of a film-substrate combination are available. Recently, a general-purpose program for the optical characterization of thin films in multilayers was presented. ${ }^{1}$ The main feature of this software is its capacity for linking the optical parameters of the layers of several different samples. The underlying idea is that each layer of a multilayer structure is defined by its physical thickness and a set of parameters (real numbers) that model its complex refractive index (for instance, the real numbers corresponding to a dispersion formula). Thus, the whole multilayer structure is fully determined with a set of real values: the thickness and some dispersion parameters for each layer (plus the values for the substrate). Usually, several of these parameters are well known (e.g., the optical constants and the thickness of the substrate), whereas others are only approximate and are the $m$ unknowns $P_{i}(i=1, \ldots, m)$ that are inferred by the computer program. This

J. Sancho-Parramon, J. Ferré-Borrull, and S. Bosch are with the Department Física Aplicada i Optica, Universitat de Barcelona, Diagonal 647, 08028 Barcelona, Spain. M. C. Ferrara is with Unità Tecnico Scientifica Materiali Compositi e Nanostrutturati ENEA Centro Ricerche Brindisi, Strada Statale 7 Appia km 713, 7, 72100 Brindisi, Italy.

Received 26 June 2002; revised manuscript received 3 October 2002.

0003-6935/03/071325-05\$15.00/0

(C) 2003 Optical Society of America program always requires a merit function to evaluate the consistency of computed and experimental data. The chi-square estimator $\left(\chi^{2}\right)$ is an extremely versatile function because it may be defined for several samples and various kinds of measured data taken together. Suppose the experimental data consist of $n$ measurements $y_{i}(i=1, \ldots, n)$ with associated estimated errors $\sigma_{i}(i=1, \ldots, n)$ corresponding to independent variables $x_{i}$ (for instance, reflectance values $y_{i}$ for a range of wavelengths $x_{i}$ ). Standard thin-film calculation methods ${ }^{2}$ enable us to compute the data corresponding to our model stack $\left[y\left(x_{i}\right)\right]$ in terms of the unknown parameters $P_{i}(i=1, \ldots, m)$. Then,

$$
\chi^{2}\left(P_{1}, \ldots, P_{m}\right)=\frac{1}{n-m-1} \sum_{i=1}^{n}\left[\frac{y_{i}-y\left(x_{i}\right)}{\sigma_{i}}\right]^{2},
$$

where $n$ is the total number of available measurements and $m$ is the number of unknown parameters. In the case of a simultaneous characterization of different (say $q$ ) samples, ${ }^{3}$ the measurements are $y_{i}^{j}(i=$ $1, \ldots, n ; j=1, \ldots, q)$ and the merit function is

$$
\begin{gathered}
\chi^{2}\left(P_{1}^{1}, \ldots P_{m 1}^{1}, P_{1}^{2}, \ldots P_{m 2}^{2}, \ldots, P_{1}^{q}, \ldots P_{m q}^{q}\right) \\
=\frac{1}{n q-m-1} \sum_{j=1}^{q} \sum_{i=1}^{n}\left[\frac{y_{i}^{j}-y^{j}\left(x_{i}\right)}{\sigma_{i}{ }^{j}}\right]^{2},
\end{gathered}
$$

where $\left(P_{1}^{1}, \ldots P_{\mathrm{m} 1}^{1}, P_{1}^{2}, \ldots P_{\mathrm{m} 2}^{2}, \ldots, P_{1}^{\mathrm{q}}, \ldots P_{\mathrm{mq}}^{\mathrm{q}}\right)$ is the set of parameters needed to describe the collection of the $q$ samples. In Eq. (2) the total number of unknown parameters is $m=m_{1}+\ldots+m_{q}\left(P_{1}{ }^{1}, \ldots\right.$, $P_{m 1}^{1}$ corresponding to sample $1, P_{1}^{2}, \ldots P_{m 2}^{2}$ corre- 
sponding to sample $2, \ldots$ ), and the superindex $j$ indentifies the sample. This mathematical approach easily allows for linking together several parameters, forcing them to vary as if they were one. This approach will be the basis for our global fitting procedure, which is described in subsection 2.B. In summary, the $m$ unknown parameters shown in Eq. (2) are considered independent (if several parameters are linked together, only one is independent). For simplicity in Eq. (2) we assume the same number $n$ of measured data for each one of the samples; if this is not the case, an equivalent formula with internal summations up to different values $n_{q}$ should be used.

Note that this procedure is conceptually different from an individual characterization for each sample, because here all available data for all samples are considered in a single optimization procedure. In the case of a lot of layers and samples, the number of parameters $m$ grows dramatically, and the inversion problem is difficult to solve. Nevertheless, the number of parameters is often greatly reduced by the introduction of realistic considerations based on manufacturing information. For instance, in the case of filters with only two materials [with high $(\mathrm{H})$ and low (L) refractive indexes), it can be assumed that all the $\mathrm{H}$ as well as $\mathrm{L}$ layers have the same refractive index. Similarly, restrictions can be included when several samples are characterized simultaneously. For example, layers deposited during the same physical process have the same refractive index but may have different thicknesses. The restrictions may cover a wide range of possibilities, and the user, according to the knowledge of the production process, must decide what conditions should reasonably be imposed. The uncertainty of the final parameters is calculated through well-known numerical methods.1,4

In the present study we use this kind of procedure for the optical characterization of multilayer structures by means of near-normal reflectance measurements only. The manufacturing information is used to identify which layers in the samples should have the same thickness, the same refractive index, or both, thus reducing the number of parameters. We show that multiple-sample simultaneous fitting is essential if there are to be meaningful results in our practical situation.

\section{Case Study}

We illustrate our procedures by considering four samples $(\mathrm{A}, \mathrm{B}, \mathrm{C}$, and D) that are antireflection coated (in the visible range) by three-layer designs at both sides of soda lime glass substrates ( $4 \mathrm{~mm}$ thick). Figure 1 shows a sketch of the structure. Stacks of L-, medium- (M), and H-index layers were manufactured by means of dip-coating films with the adequate material composition. Silica, and two mixed-oxide silica-titania sols, with a nominal molar ratio $\mathrm{Si} / \mathrm{Ti}=$ $1 / 1$ and $\mathrm{Si} / \mathrm{Ti}=1 / 9$ were used to produce the layers of $\mathrm{L}, \mathrm{M}$, and $\mathrm{H}$ refractive indices, ${ }^{5}$ respectively. Dip coating is a process in which the substrate to be coated is immersed in liquid and then withdrawn with a well-defined withdrawal speed under con-

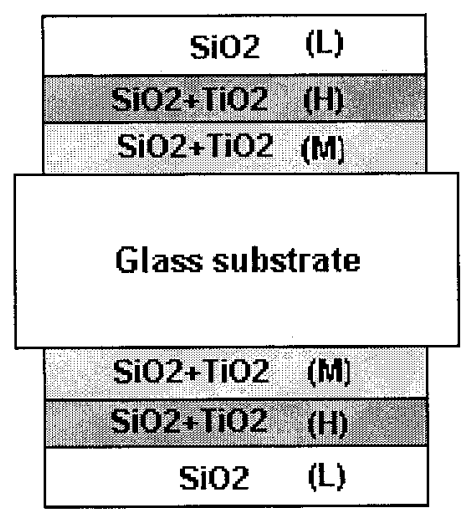

Fig. 1. Sketch of the multilayer configuration.

trolled temperature and atmospheric conditions. The layer thickness is mainly defined by the withdrawal speed and also by the solid content and viscosity of the liquid. ${ }^{6}$ Thus, both sides of the sample should have identical corresponding layers whose thicknesses depend on the withdrawal speed. Table 1 summarizes the withdrawal speeds (in centimeters per minute) for the different materials in the various samples.

Reflectances at near-normal incidence were measured with a VARIAN Cary 5 spectrophotometer in the range of 380-780 $\mathrm{nm}$. The estimated error in this range is approximately 0.003 . The refractive index of the layers was modeled with a two-term Cauchy expression with no absorption, according to the formulas

$$
n(\lambda)=n_{0}+\frac{n_{1}}{\lambda^{2}}, \quad k(\lambda)=0 .
$$

The refractive index of the substrate was previously determined from reflectance and transmittance measurements.

\section{A. Sample-by-Sample Characterization}

It is always assumed that layers made of the same material have (at both sides) the same refractive index and thickness. Thus, nine parameters are needed to determine any of the four samples: thickness plus two dispersion parameters $\left(n_{0}, n_{1}\right)$ for each material, multiplied by the three different materials. Thus, a set of 36 parameters describes the four samples. Table 2 summarizes the results of this procedure. Figure 2 compares the refractive-index profiles. Three points arising from these results should be highlighted. First, uncertainty in the de-

Table 1. Withdrawal Speeds for Obtaining the Layers of the Samples A-D

\begin{tabular}{cccc}
\hline Sample & $\mathrm{L}(\mathrm{cm} / \mathrm{min})$ & $\mathrm{H}(\mathrm{cm} / \mathrm{min})$ & $\mathrm{M}(\mathrm{cm} / \mathrm{min})$ \\
\hline A & 20 & 25 & 18 \\
B & 20 & 25 & 23 \\
C & 20 & 20 & 18 \\
D & 20 & 15 & 23 \\
\hline
\end{tabular}


Table 2. Calculated Parameters for the Samples A-D Obtained by a Sample-by-Sample Characterization

\begin{tabular}{|c|c|c|c|c|c|c|c|c|}
\hline Sample & Material & $d(\mathrm{~nm})$ & $\delta d(\mathrm{~nm})$ & $n_{0}$ & $\delta n_{0}$ & $n_{1}\left(\mathrm{~nm}^{2}\right)$ & $\delta n_{1}\left(\mathrm{~nm}^{2}\right)$ & $\chi^{2}$ \\
\hline \multirow[t]{3}{*}{ A } & $\mathrm{L}$ & 104.1 & 6.3 & 1.49 & 0.20 & 4995. & 45105 & 0.16 \\
\hline & $\mathrm{H}$ & 89.2 & 9.4 & 1.85 & 0.19 & 49825 . & 16211 & \\
\hline & $\mathrm{M}$ & 83.6 & 8.3 & 1.70 & 0.08 & 30119. & 7008 & \\
\hline \multirow[t]{3}{*}{ B } & $\mathrm{L}$ & 104.6 & 2.9 & 1.51 & 0.03 & 4117 & 5384 & 0.17 \\
\hline & $\mathrm{H}$ & 89.1 & 1.4 & 1.83 & 0.10 & 56214 & 16130 & \\
\hline & $\mathrm{M}$ & 93.3 & 2.3 & 1.68 & 0.02 & 30590 & 5543 & \\
\hline \multirow[t]{3}{*}{$\mathrm{C}$} & $\mathrm{L}$ & 108.0 & 2.6 & 1.47 & 0.04 & 4976 & 12095 & 0.14 \\
\hline & $\mathrm{H}$ & 81.4 & 0.3 & 1.79 & 0.04 & 49078 & 10313 & \\
\hline & $\mathrm{M}$ & 84.5 & 1.9 & 1.67 & 0.03 & 30737 & 6978 & \\
\hline \multirow[t]{3}{*}{ D } & $\mathrm{L}$ & 106.7 & 2.2 & 1.48 & 0.04 & 4923 & 15839 & 0.11 \\
\hline & $\mathrm{H}$ & 69.3 & 1.8 & 1.79 & 0.03 & 50569 & 7820 & \\
\hline & $\mathrm{M}$ & 94.9 & 2.8 & 1.67 & 0.02 & 30616 & 9799 & \\
\hline
\end{tabular}

termination of the parameters is high, which is mainly due to the limited precision of the reflectance measurements. Second, the final values of the merit function $\left(\chi^{2}\right)$ are unrealistically low (owing to the high number of free parameters in the optimization process); but one should remember that all the numerical fittings with $\chi^{2}<1$ are within the margins of experimental error.1,4 Third, the results for layers deposited in equivalent conditions are not all similar (compare, for example, the differences among the $\mathrm{L}$ layers in Table 2, even when all these layers for the four samples are being obtained at the same withdrawal speed).

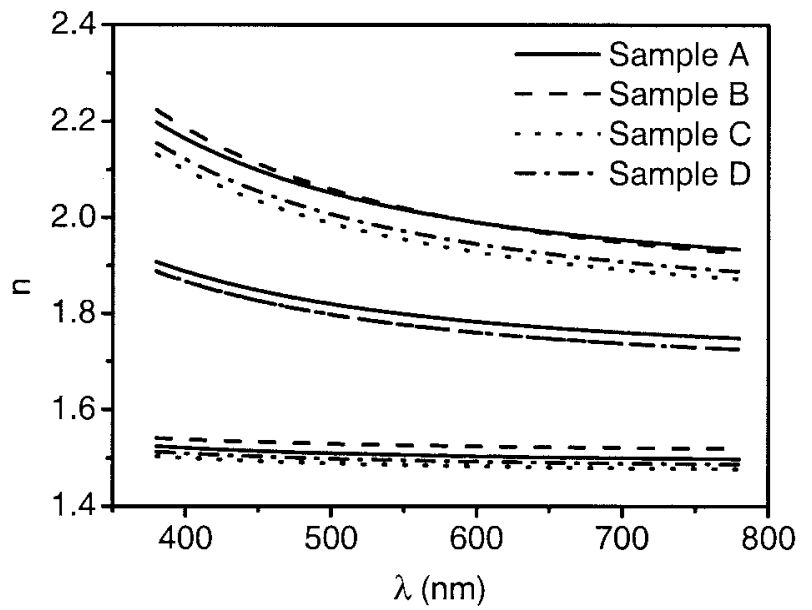

Fig. 2. Calculated dispersions of the three materials for the samples A-D.

\section{B. Simultaneous Four-Sample Characterization}

We now consider each material that has a fixed composition and is always deposited under the same highly controlled deposition conditions to have a welldefined optical dispersion, and the differences in thicknesses are associated with differences in withdrawal speed. For purposes of characterization, a change in any experimental detail in sol composition and viscosity, withdraw temperature or atmospheric conditions, and thermal curing is equivalent to a change in optical dispersion, as if we were dealing with a truly different material. Now the number of parameters to define the four samples is greatly reduced. Only three are needed for describing the $\mathrm{L}$ layers, since by manufacture the layers are equivalent even in thickness because of being obtained with the same withdrawal speed (see column L in Table 1). For the $\mathrm{H}$ layers, five parameters are needed: two for optical dispersion and three for the thicknesses, corresponding to three withdrawal speeds (see column $\mathrm{H}$ in Table 1). Finally, four parameters are needed for the M layers, since the layers have the same optical dispersion, but two thicknesses (column $\mathrm{M}$ in Table 1). This amounts to 12 parameters in total $(m=12)$. Table 3 summarizes the results of this fitting procedure. Figure 3 compares the refractive indices, and Fig. 4 compares the results in terms of computed reflectances for sample D. The experimental data are shown together with the computed data obtained by means of the individual fitting (where the final merit function was $\chi^{2}=0.11$, the lowest value obtained) and with the computed data obtained by means of the global fitting (where the

Table 3. Calculated Parameters by a Simultaneous Four-Sample Characterization

\begin{tabular}{|c|c|c|c|c|c|c|c|c|}
\hline Material & Withdrawal Speed & $d(\mathrm{~nm})$ & $\delta d(\mathrm{~nm})$ & $n_{0}$ & $\delta n_{0}$ & $n_{1}\left(\mathrm{~nm}^{2}\right)$ & $\delta n_{1}\left(\mathrm{~nm}^{2}\right)$ & $\chi^{2}$ \\
\hline $\mathrm{L}$ & 20 & 105.9 & 0.8 & 1.493 & 0.017 & 4491. & 5079. & 0.89 \\
\hline \multirow[t]{3}{*}{$\mathrm{H}$} & 15 & 67.4 & 0.5 & 1.879 & 0.020 & 34557. & 2731. & \\
\hline & 20 & 81.8 & 0.2 & & & & & \\
\hline & 25 & 91.1 & 0.4 & & & & & \\
\hline \multirow[t]{2}{*}{$\mathrm{M}$} & 18 & 84.1 & 0.6 & 1.698 & 0.008 & 29327. & 1620 . & \\
\hline & 23 & 96.3 & 0.7 & & & & & \\
\hline
\end{tabular}




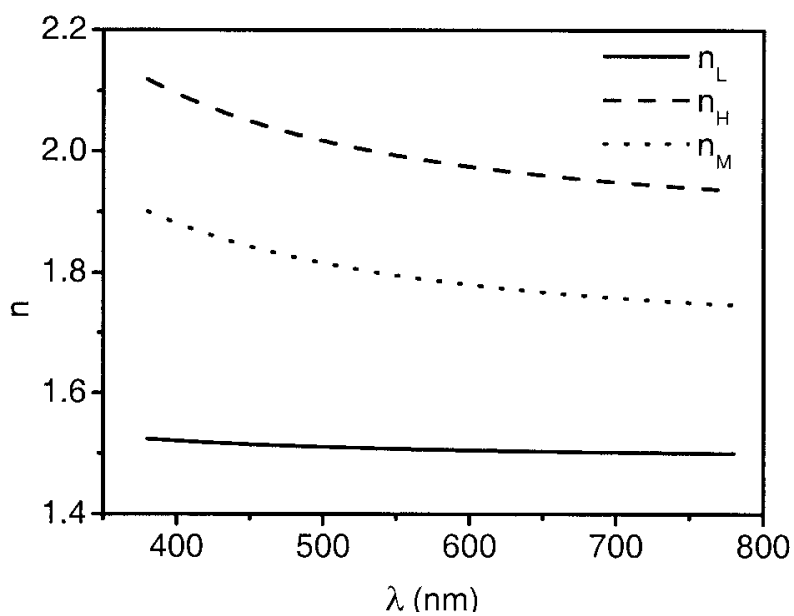

Fig. 3. Calculated dispersions of the three materials in the global fitting.

final merit function was $\chi^{2}=0.89$ ). Regarding the three comments on the previous sample-by-sample characterization, we now note that (i) the uncertainty in the parameters is greatly reduced, (ii) the final value of the merit function is higher (although still $\chi^{2}$ $<1$ ), and (iii) each component material is associated with a single refractive index.

\section{Discussion}

Two global strategies for the optical characterization of multilayer samples, by means of near-normal reflectance measurements only, are compared above. To assess the methods' practical validity, it is necessary now to analyze their physical and numerical implications. Experimental evidence corroborates our conclusions.

The normal practice in thin-films research is the sample-by-sample characterization. There are in principle no objections to this procedure. However, in our case there are at least two reasons for great

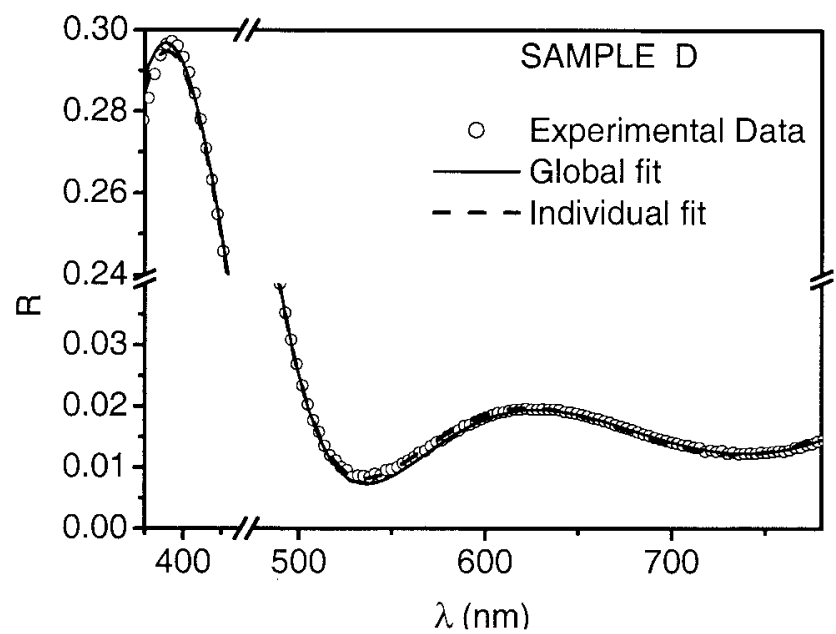

Fig. 4. Comparison among the measured (experimental) data, the individual fit of sample $\mathrm{D}\left(\chi^{2}=0.11\right)$, and the corresponding global fitting $\left(\chi^{2}=0.89\right)$. caution in analyzing these results: the measurements are not very precise, and the samples have many layers (a lot of parameters to be determined). The combination of these two points leads to a final merit function that is low, but whose parameters are quite inaccurate. On the contrary, the simultaneous optimization procedure leads to a higher final merit function with less inaccuracy in parameters. It is also very important to remember that the final value of the merit function $\chi^{2}$ gives an indication of the quality of the fit. In short, $\chi^{2} \sim 1$ indicates that the fit is as good as can be, given the accuracy of the data. Thus, even the best figure of merit of the individual fits $\left(\chi^{2}=0.11\right.$ in Table 1$)$ should not be judged physically as much better than the $\chi^{2}=0.89$ obtained for the simultaneous four-sample fitting. We can equivalently look at the problem in another way: The results shown in Tables 2 and 3 are correct since they are compatible when the uncertainties are included; however, only the results in Table 3 lead to a meaningful (i.e., useful in a practical sense) optical characterization of the materials.

This last statement is clearly demonstrated when one tries to refine the designs. Our laboratory tests confirmed that using the optical data obtained from individual sample fits to try to refine a design (making minor adjustments in thickness by changing the withdrawal speed), leads to meaningless results. These results demonstrate that the determination of the optical parameters is inaccurate, since we do not get the expected change from our adjustment. This fact should be foreseen simply by looking at the dispersion curves in Fig. 2; the visible differences give rise to very different reflectance performances when the six layers are added together. However, the optical data from the global fit are indeed valid for a refinement procedure. This is (in our opinion) the final justification for the procedure we recommend: the global one. No particular example needs to be shown; essentially, optical data inferred from individual fits do not allow refinement of the designs, whereas data deduced from the global fitting do.

\section{Conclusion}

For cases in which the optical characterization of multilayers is very difficult because of the inherent inaccuracy of spectral data and the high number of parameters to be determined, a global optimization procedure is proposed. The key point of the method is the simultaneous use of all available spectral data and of the information on the manufacture of the samples in a single optimization procedure.

M. C. Ferrara thanks Saverio Mazzarelli for collaboration in the manufacture of the samples.

\section{References}

1. S. Bosch, J. Ferré-Borull, and J. Sancho-Parramon, "A general purpose software for optical characterisation of thin films: Specific features for microelectronic applications," Solid-State Electron. 45, 703-709 (2001). 
2. H. A. Macleod, Thin Film Optical Filters (Wiley, New York, 1976).

3. S. Bosch, N. Leinfellner, E. Quesnel, A. Duparré, J. FerréBorrull, S. Guenster, and D. Ristau, "New procedure for the optical characterization of high-quality thin films," in Optical Metrology Roadmap for the Semiconductor, Optical, and Data Storage Industries, G. A. Al-Jumaily, A. Duparré, and B. Singh, eds., Proc. SPIE 4099, 124-130 (2000).

4. W. H. Press, S. A. Teukolsky, W. T. Vetterling, and B. P. Flan- nery, Numerical Recipes in $C$ (Cambridge University Press, New York, 1992).

5. M. C. Ferrara, S. Mazzarelli, J. Sancho-Parramon, and S. Bosch. "Sol-gel process for the production of large area coatings," presented at the III Workshop Italiano Sol-Gel, Trento, Italy, 13-14 June 2002.

6. C. J. Brinker and G. W. Scherer, The Physics and the Chemistry of Sol-gel Processing (Academic, New York, 1990). 